\title{
Smooth incidence maps give valuable insight into $Q$ fever outbreaks in The Netherlands
}

\author{
Wim van der Hoek ${ }^{1}$, Jan van de Kassteele ${ }^{2}$, Ben Bom ${ }^{2}$, Arnout de Bruin ${ }^{1}$, Frederika Dijkstra ${ }^{1}$, \\ Barbara Schimmer ${ }^{1}$, Piet Vellema ${ }^{3}$, Ronald ter Schegget ${ }^{4}$, Peter M. Schneeberger ${ }^{5}$ \\ ${ }^{1}$ Centre for Infectious Disease Control, National Institute for Public Health and the Environment, PO Box 1, \\ 3720 BA Bilthoven, The Netherlands; ${ }^{2}$ Department of Statistics, Mathematical Modelling and Data Logistics, \\ National Institute for Public Health and the Environment, PO Box 1, 3720 BA Bilthoven, The Netherlands; \\ ${ }^{3}$ Department of Small Ruminant Health, Animal Health Service, PO Box 9, 7400 AA Deventer, The \\ Netherlands; " Municipal Health Service "Brabant Zuidoost", Stadhuisplein 2, 5611 EM Eindhoven, The \\ Netherlands; ${ }^{5}$ Department of Medical Microbiology and Infection Control, Jeroen Bosch Hospital, PO Box \\ 90153, 5200 ME 's Hertogenbosch, The Netherlands
}

\begin{abstract}
From 2007 through 2009, The Netherlands faced large outbreaks of human Q fever. Control measures focused primarily on dairy goat farms because these were implicated as the main source of infection for the surrounding population. However, in other countries, outbreaks have mainly been associated with non-dairy sheep and The Netherlands has many more sheep than goats. Therefore, a public discussion arose about the possible role of non-dairy (meat) sheep in the outbreaks. To inform decision makers about the relative importance of different infection sources, we developed accurate and high-resolution incidence maps for detection of Q fever hot spots. In the high incidence area in the south of the country, full postal codes of notified Q fever patients with onset of illness in 2009, were georeferenced. Q fever cases $(\mathrm{n}=1,740)$ were treated as a spatial point process. A $500 \times 500 \mathrm{~m}$ grid was imposed over the area of interest. The number of cases and the population number were counted in each cell. The number of cases was modelled as an inhomogeneous Poisson process where the underlying incidence was estimated by 2-dimensional P-spline smoothing. Modelling of numbers of Q fever cases based on residential addresses and population size produced smooth incidence maps that clearly showed Q fever hotspots around infected dairy goat farms. No such increased incidence was noted around infected meat sheep farms. We conclude that smooth incidence maps of human notifications give valuable information about the Q fever epidemic and are a promising method to provide decision support for the control of other infectious diseases with an environmental source.
\end{abstract}

Keywords: Q fever, smooth incidence maps, dairy goats, The Netherlands.

\section{Introduction}

Q fever is a zoonotic infectious disease with worldwide occurrence that manifests in humans primarily as an acute febrile syndrome, pneumonia or hepatitis. There is an extensive animal reservoir, but transmission to humans of the causative bacterium, Coxiella burnetii, is mainly associated with ruminants (goats, sheep and cattle). Most infected animals show no clinical signs but the infection can cause late abortion, especially in goats and sheep (Roest et al., 2011). From 2007 through 2009, the south of The

Corresponding author:

Wim van der Hoek

Centre for Infectious Disease Control

National Institute for Public Health and the Environment

PO Box 1, 3720 BA Bilthoven, The Netherlands

Tel +31 30274 2074; Fax +31 302744409

E-mail: wim.van.der.hoek@rivm.nl
Netherlands faced large outbreaks of human Q fever (Schimmer et al., 2008, 2009). A clear epidemiological link was established with dairy goat farms, and to a lesser extent with dairy sheep farms, that experienced high abortion rates (abortion waves) caused by C. burnetii. During abortion or parturition of infected goats and sheep, billions of C. burnetii end up in the environment. Contaminated dust particles can travel by air, depending on wind and weather conditions, vegetation and soil characteristics (Astobiza et al., 2011; van der Hoek et al., 2011). People living in close proximity $(<5 \mathrm{~km})$ to a farm with a high animal abortion rate run a much higher risk of $\mathrm{Q}$ fever than people living further away (Schimmer et al., 2010). The veterinary control measures implemented in The Netherlands have therefore focused on dairy goat farms and dairy sheep farms and included mandatory mass vaccination, culling of pregnant animals on infected farms and hygiene measures (van der Hoek et al., 2010; Roest et al., 2011). 
In addition to infections that occur from ambient exposure at some distance from a major source, small outbreaks can also occur when people visit an infectious source and are in close contact with infected animals, e.g. non-dairy sheep (Porten et al., 2006; Koene et al., 2011; Whelan et al., 2012). In fact, most Q fever outbreaks in other countries have been associated with sheep, rather than goats. For example, in Germany in 2005, 331 Q fever cases, 160 of which were confirmed by laboratory testing, occurred in a residential area close to a meadow with C. burnetii-infected sheep (Gilsdorf et al., 2008). When the municipal health service (MHS) “Brabant Zuidoost” reported that $46 \mathrm{Q}$ fever cases had visited a meat sheep farm in FebruaryMarch 2009, a nationwide discussion started about the possible role of non-milk producing farms, including small hobby farms and petting zoos that were not included in control measures. An unknown number of commercial meat sheep farms, petting zoos and free ranging sheep flocks were tested at request of the local MHS, municipal authorities or the owners of the animals. This resulted in a total of 21 locations that tested positive for C. burnetii DNA with polymerase chain reaction (PCR) on vaginal swabs. An expert group headed by the director of the Centre for Infectious Disease Control of The Netherlands was commissioned to advise the Ministry of Health and the Ministry of Agriculture about the possible role of nonmilk producing farms in the ongoing $Q$ fever outbreaks.

Human-to-human transmission of C. burnetii plays a minor role and any geographical clustering of human cases therefore suggests a common environmental point source. To assess to what extent different animal sources contribute to the transmission risk for the surrounding population and to visualise the findings for policy makers, we developed accurate and high-resolution incidence maps for detection of $Q$ fever hot spots.

\section{Materials and methods}

\section{Human Q fever cases}

In The Netherlands, medical microbiology laboratories and attending physicians are both legally required to report every diagnosis of acute $\mathrm{Q}$ fever to the local MHS. The MHS uses a short trawling questionnaire to ask the patient for possible occupational or recreational exposure. Limited anonymous information on each case that fulfils the notification criteria, i.e. a presentation with fever, or pneumonia, or hepatitis, and with a laboratory confirmation, is entered into the electronic national infectious diseases surveillance database. For the present study, the MHS provided the full postal code of the home address and date of onset of illness of all patients that had been notified with acute $\mathrm{Q}$ fever as a separate anonymous file.

\section{Infected dairy goat and dairy sheep farms}

From 2005 through 2009, 28 dairy goat farms and two dairy sheep farms were confirmed with abortion waves caused by C. burnetii resulting in abortion rates of up to $60 \%$ (Roest et al., 2011). Information on these farms was provided by the Animal Health Service. A system of mandatory bulk tank milk monitoring was initiated in October 2009 and by 1 January 2010, 61 farms (all dairy goat farms) had tested positive. The addresses of bulk tank milk positive farms were publicly available on the website of the Food and Consumer Product Safety Authority (http://www. vwa.nl/onderwerpen/dierziekten/dossier/q-koorts/ kaart-met-overzicht-van-besmette-bedrijven).

\section{Suspected non-dairy sheep farms}

In 2009, the MHS “Brabant Zuidoost” received 379 notifications of acute $Q$ fever. It was assumed that the majority of these cases were explained by proximity of residence to a dairy-goat farm (Fig. 1, farm A) that had experienced a C. burnetii-induced abortion wave in 2008. The situation in 2008 around this farm has been described in detail (Schimmer et al., 2010). In 2009 the same dairy goat farm did not experience an unusually high abortion rate but 30 of 30 goats tested positive with PCR on vaginal swabs. In addition 10 of 10 surface swabs, six out of six air samples, and one of four manure samples tested positively for C. burnetii DNA by PCR at this farm. This application of the PCR technique has been described elsewhere (de Bruin et al., 2011).

In the MHS trawling questionnaire, 46 out of the $379 \mathrm{Q}$ fever patients indicated that they had visited a meat sheep farm (Fig. 1, farm B), located about $10 \mathrm{~km}$ southwest from farm A. Farm B with >1,500 sheep had opened for the public on weekends during the lambing season in February and March 2009. The owner estimated that a total of 12,000 people visited the farm during this 2-month period. Visitors had the opportunity to witness lambing and to bottle-feed the newborn lambs. Investigations by the Food and Consumer Product Safety Authority established that the farm had a normal lambing season with a low 


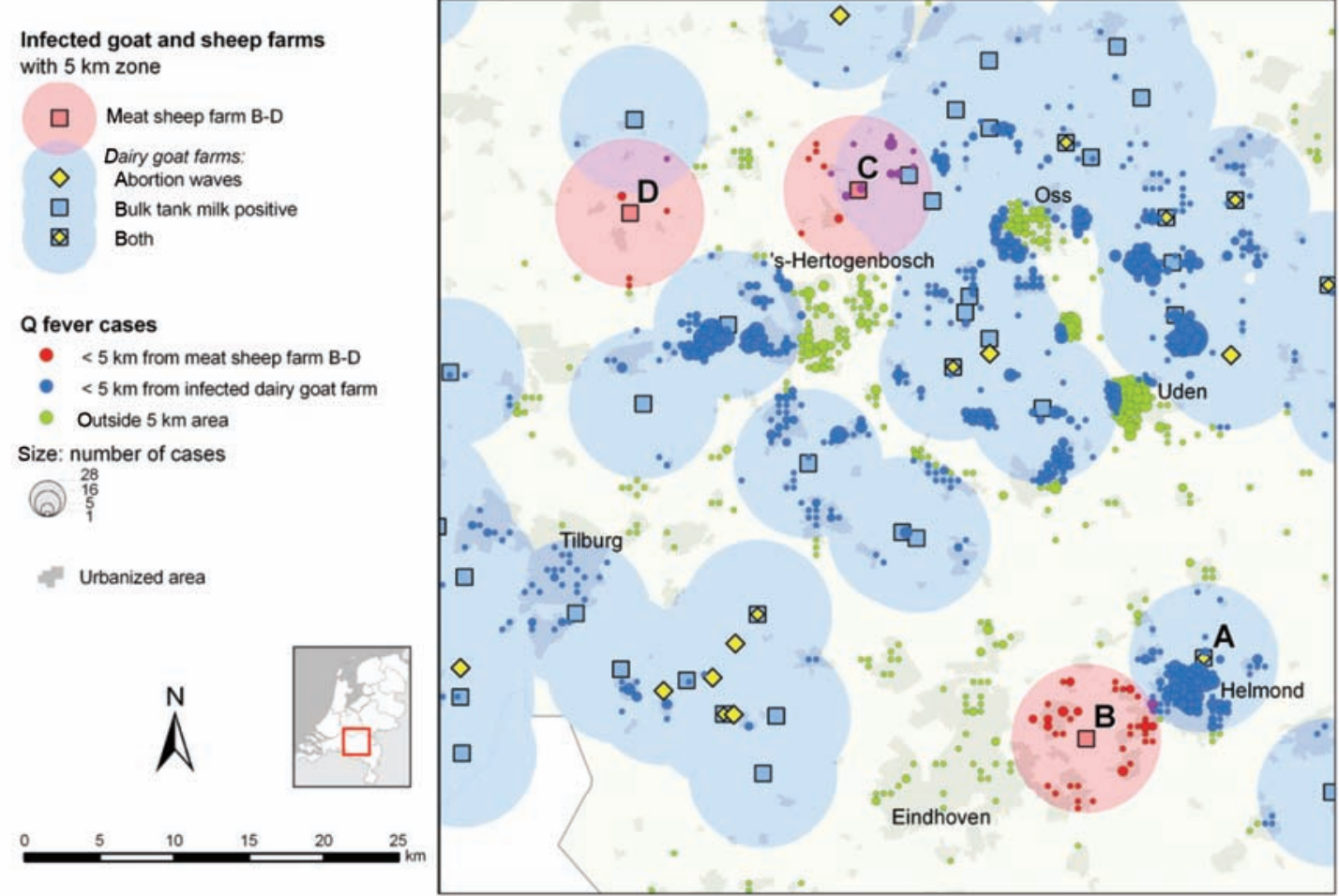

Fig. 1. Map of the study area with human Q fever cases and infected goat and sheep farms in 2009. Five km areas around infected farms are shown in blue (infected dairy goat farms) or red (meat sheep farm B-D). The residential addresses of human cases are indicated by blue dots or red dots if located within such a $5-\mathrm{km}$ zone, or by green dots if outside the $5-\mathrm{km}$ zones. Notifications include all cases with day of onset of illness in 2009 ( $\mathrm{n}=1,740)$.

abortion rate (three abortions on 1,200 lambings). Twenty sheep were tested (PCR on vaginal swabs) in May 2009 of which 19 were positive. In addition, seven out of eight air samples around the farm were positive for C. burnetii DNA.

Farm C (Fig. 1) was a meat sheep farm with 1,000 animals. The local MHS suspected a relation with 22 human $\mathrm{Q}$ fever cases in the same municipality. There had been no abortion problems and the farm had an enclosed stable for lambing and no access to the public. PCR results of vaginal swabs in August 2009 were ambiguous for six animals and negative for 14 animals. Two dust samples were taken of which one was positive and one ambiguous. In January 2010, another 20 sheep were sampled, with two positive findings, one ambiguous and 17 negative results.

Farm D (Fig. 1) was used for trading meat sheep and therefore had a high turnover of animals. There had been 17 human $\mathrm{Q}$ fever cases in the municipality where this farm was located. One of 20 sheep from this farm tested positive with PCR on vaginal swabs.

\section{Data analysis}

Analysis focused on a $60 \times 60 \mathrm{~km}$ area in the south of the Netherlands, encompassing farms A-D (Fig. 1).
The home addresses of all 1,740 Q fever cases in this area with an onset of illness in 2009 were geo-referenced in an $\mathrm{X} / \mathrm{Y}$ coordinate system based on the centroid of their 6-position postal code (generally part of a street). There are about 452,000 6-position postal code areas in The Netherlands, which provides a high resolution geographically. Farms with C. burnetiiinduced abortion waves and farms that tested positive in bulk tank milk monitoring were georeferenced based on their full address (6-position postal code, street and house number). Population data at the 6position postal code level were obtained from the Dutch Land Registry Office and Statistics Netherlands.

\section{Development of smooth incidence maps}

The occurrence of $\mathrm{Q}$ fever cases in the area was treated as an inhomogeneous Poisson process, or Cox process, where the intensity varies in space (Lawson, 2006). To estimate this intensity we first imposed a fine regular grid over the area of interest. We chose cell sizes of $500 \times 500 \mathrm{~m}$, resulting in a large 2-dimensional grid with $m_{1}$ cells in the X-direction and $m_{2}$ cells in the Y-direction. Each cell contains counts of the number of $\mathrm{Q}$ fever cases and population numbers. 
Because of the small cell size, the observed incidences fluctuate strongly. We believe that the true incidence surface varies smoothly in space. We applied 2-dimensional P-spline smoothing to estimate the underlying incidence surface. The idea behind P-splines is to build a large B-spline basis with equally spaced knots and, as a restriction, penalise large changes in the parameter values between neighbouring parameters (secondorder differences) (Eilers and Marx, 1996).

The model is written as follows. The data vector $y$ consists of the elements of the large grid with counts arranged in a vector of length $m_{1} m_{2}$, with the first dimension varying the fastest. These are Poisson distributed with intensity vector $\mu$. The intensity is the product of the offset (population numbers in each cell, vector $E$ ) and a smooth incidence surface, represented by $\exp (\eta)$. Vector $\eta$ is the inner product of a large 2dimensional B-spline basis matrix (size $\left.m_{1} m_{2} \times n_{1} n_{2}\right)$ and parameter vector $\theta$ of length $n_{1} n_{2}$. The parameters are found by penalised iterative reweighted least squares using the scoring algorithm (Wood, 2006). For a certain value of the smoothing parameter $\lambda$ and initial value for $\mu$ the algorithm is as follows:

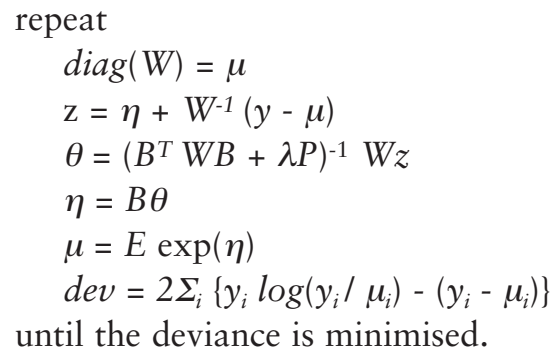

The 2-dimensional B-spline basis matrix $B$ is found by calculating the Kronecker product of two 1-dimensional B-spline basis matrices, $B=B_{2} \otimes B_{1}$ (Currie et al., 2006). Matrix $B_{1}$ (size $m_{1} \times n_{1}$ ) contains B-spline basis functions along the first dimension, while $B_{2}$ does this for the second dimension. Usually $n_{1}$ and $n_{2}$ are chosen large (20 to 30 ) to preserve enough flexibility (Eilers and Marx, 1996). The basis functions are easily calculated by truncated power functions (Eilers and Marx, 2010).

Matrix $P\left(\right.$ size $\left.n_{1} n_{2} \times n_{1} n_{2}\right)$ is the penalty matrix, which is calculated by the equation:

$$
P=I_{n 2} \otimes D_{1}^{T} D_{1}+D_{2}^{T} D_{2} \otimes I_{n 1}
$$

where $I$ is the identity matrix and $D_{1}$ a $\left(n_{1}-2\right) \times n_{1}$ second-order differencing matrix acting on the parameters along the first dimension, while similarly $D_{2}$ does this on the parameters along the second dimension
(Currie et al., 2006).

The optimal smoothing parameter $\lambda$ is found by minimizing the Akaike's information criterion, AIC = $d e v+2 d f$, for which the number of effective degrees of freedom (df) is given (see Eilers and Marx, 1996) by:

$$
d f=\operatorname{tr}\left\{\left(B^{T} W B+\lambda P\right)^{-1} B^{T} W B\right\}
$$

Once the algorithm has converged, the smooth incidences are found by calculating $\exp (\eta)$. A smooth incidence map is obtained by reshaping the vector $\exp (\eta)$ back into an $m_{1} \times m_{2}$ grid. The smooth $\mathrm{Q}$ fever incidence maps can in turn be related to infected farms.

Data were processed and analysed in the statistical software environment R (R Development Core Team, 2012) using the Matrix package (Bates and Maechler, 2012) and Maptools package (Lewin-Koh and Bivand, 2012). Mapping was done with ArcGIS 9 software (ESRI, Redlands, CA, USA).

\section{Results}

Fig. 1 is a map of the study area with the 1,740 human Q fever cases that occurred in 2009 plotted on the basis of their home addresses. It is apparent from this map that there is spatial clustering associated with the major urban centres in the study area: 's Hertogenbosch (population 105,485 in 2009), Oss (population 57,290), Uden (population 34,710), Helmond (population 87,670), Eindhoven (population 212,290) and Tilburg (population 184,490). The $5 \mathrm{~km}$ zones around infected farms cover a large part of the study area. The map shows a number of $\mathrm{Q}$ fever cases within the $5 \mathrm{~km}$ zones around the meat sheep farms B-D.

Fig. 2 shows the incidence of $\mathrm{Q}$ fever by municipality in 2009 and locations of infected farms. In this map the human cases are aggregated to administrative areas, which is unsatisfactory when relating human Q fever to the infected farms. Fig. 3 shows exactly the same area as Fig. 2 but human Q fever cases are now related to the population distribution to obtain a smooth incidence map. In the study area there are 51,425 6-position postal code areas and $>900,000$ addresses with an average distance between address and centroid of the postal code area of $60 \mathrm{~m}$. About five high-incidence (hotspot) areas are visible; all of them around Q fever infected dairy goat farms. No increased incidence is noted around the meat sheep farms B-D. Similarly, around some infected farms, even those with C. burnetii-induced abortion waves, the incidence is relatively low.

Fig. 4 is a detail of the map in Fig. 3, clearly illus- 


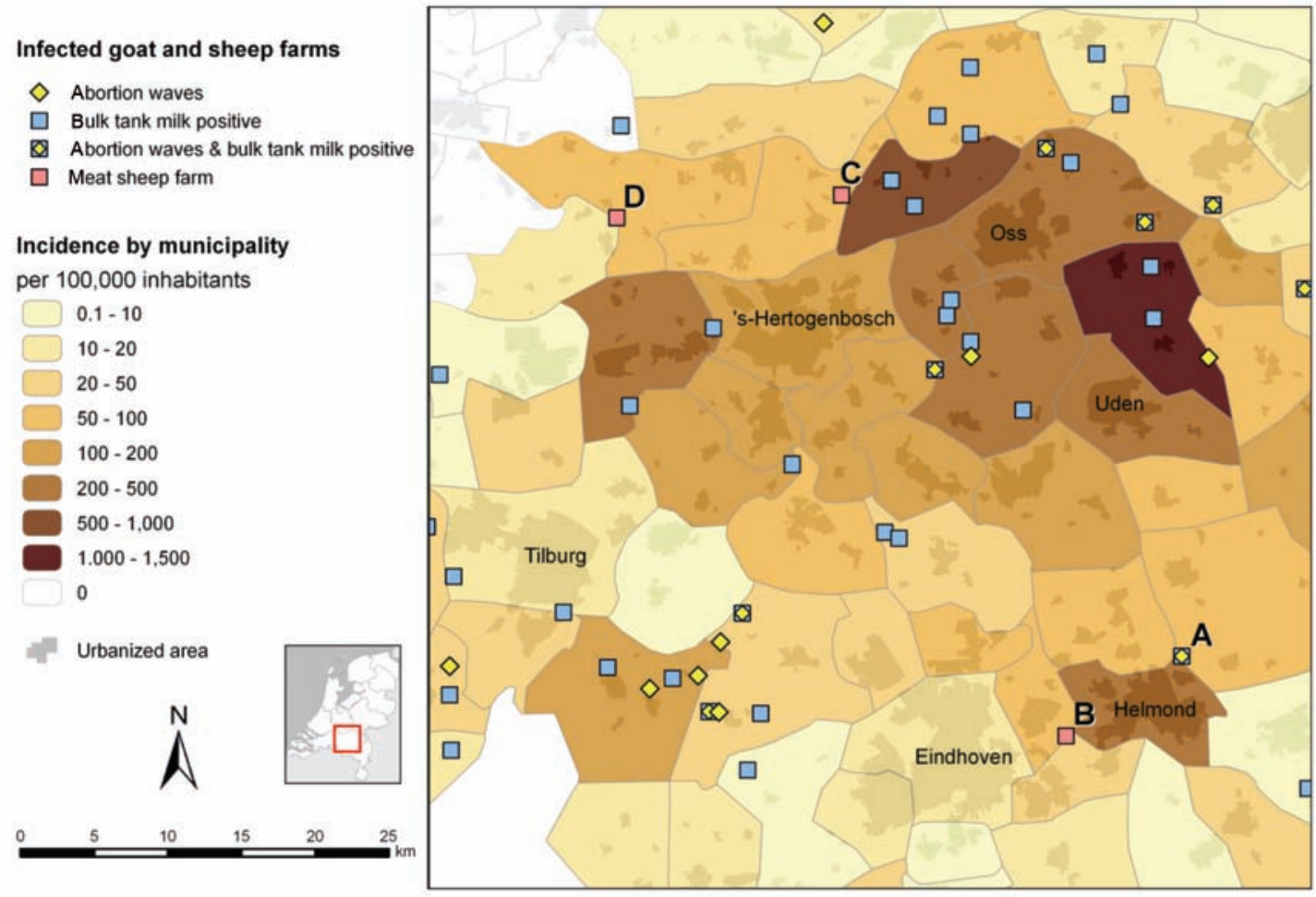

Fig. 2. Map of the study area in the southeast of The Netherlands showing incidence of human Q fever by municipality in 2009 . Incidence is based on $Q$ fever cases $(n=1,740)$ with a day of onset of illness in 2009 and population figures per January 2009.

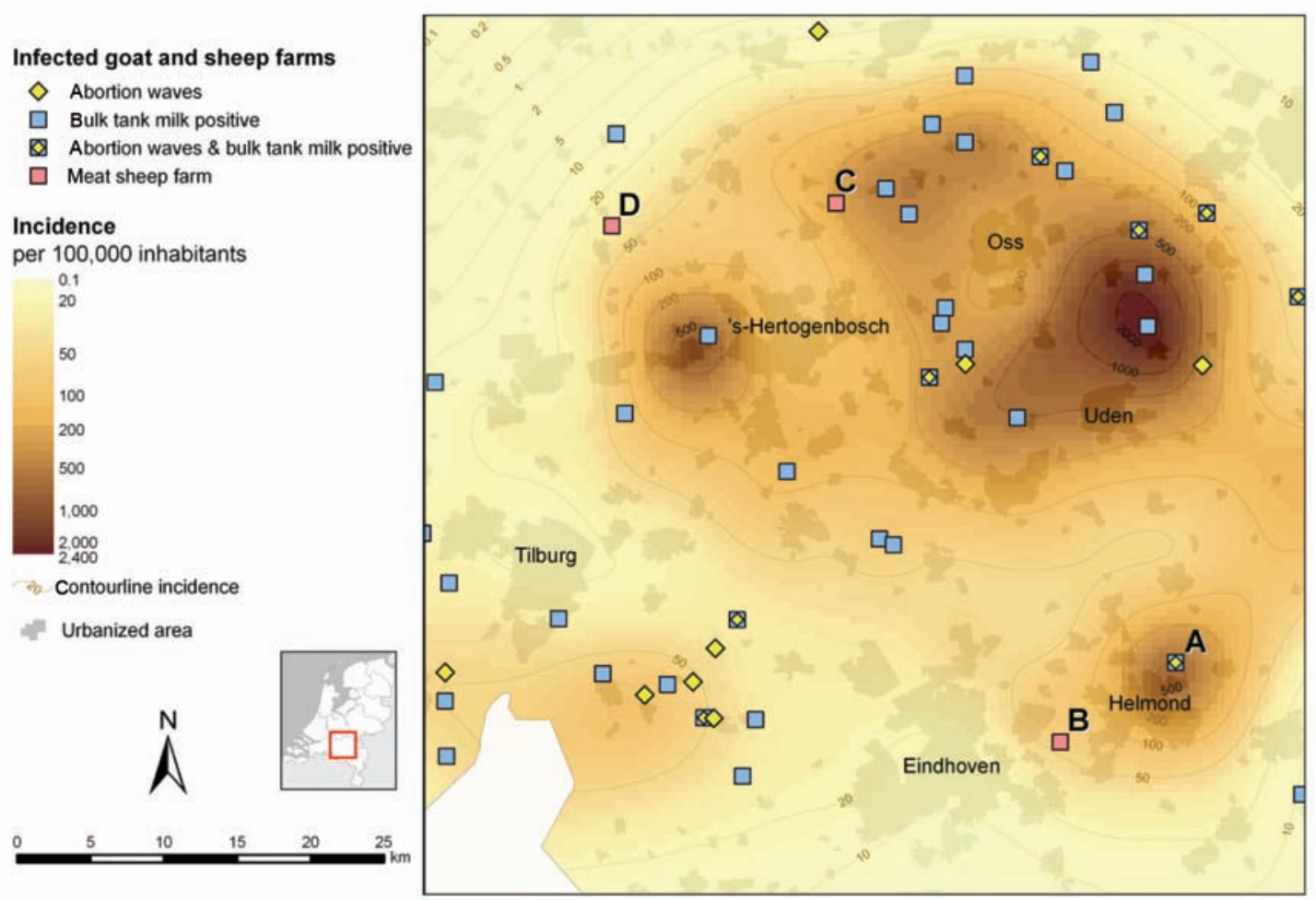

Fig. 3. Smooth incidence map of the study area. 
trating that the epicentre of the $\mathrm{Q}$ fever outbreak in that sub-region was around dairy goat farm A, while there was no increased incidence around meat sheep farm B.

\section{Discussion}

During three consecutive years, the $\mathrm{Q}$ fever epidemic in The Netherlands remained primarily in the south of the country with a clear seasonal pattern and geographical expansion with the largest number of cases occurring in 2009. Within each year, specific clusters or hot spots were observed, some of which could be linked to specific sources (Schimmer et al., 2010). Within the epidemic, in each year and area different drivers may have been responsible for infection dynamics. The contribution to the epidemic of specific farms and different types of farms and farming practices has been under debate as it was crucial for appropriate infection control measures.

In the present study, visual inspection of smooth incidence maps suggest that meat sheep farms with C. burnetii infections, unlike infected dairy goat farms, did not pose a risk for the surrounding population during the large 2009 outbreak in The Netherlands. A previous study around the dairy goat farm A, in 2008, showed a sharp decrease in Q fever incidence and risk for the population with increasing distance of house location from the farm (Schimmer et al., 2010). While dairy goat farms are clearly implicated in environmental exposure up to a distance of several $\mathrm{km}$, there is no doubt that people can get infected with C. burnetii and develop Q fever when they are in close contact with infected non-dairy sheep. A case-control study of 146 cases and 431 address-matched controls confirmed the association between a visit to meat sheep farm B of the present study (during "lamb viewing days") and Q fever disease (matched odds ratio 43, 95\% confidence interval 9-200) (Whelan et al., 2012).

Fewer than $5 \%$ of patients notified with acute Q fever in The Netherlands report an occupation in agriculture, animal care, processing or transporting of animal products (Dijkstra et al., 2012). From the available evidence it can be concluded that occupational exposure and close contact of the public with nondairy sheep can only explain a small proportion of the notified patients in The Netherlands. On the other hand, not all dairy goat farms with C. burnetiiinduced abortion waves give rise to large numbers of human Q fever cases. It has been suggested that this is due to local environmental conditions, especially vegetation density and groundwater conditions that play an important role in the transmission of C. burnetii from infected farms to humans (van der Hoek et al., 2011).

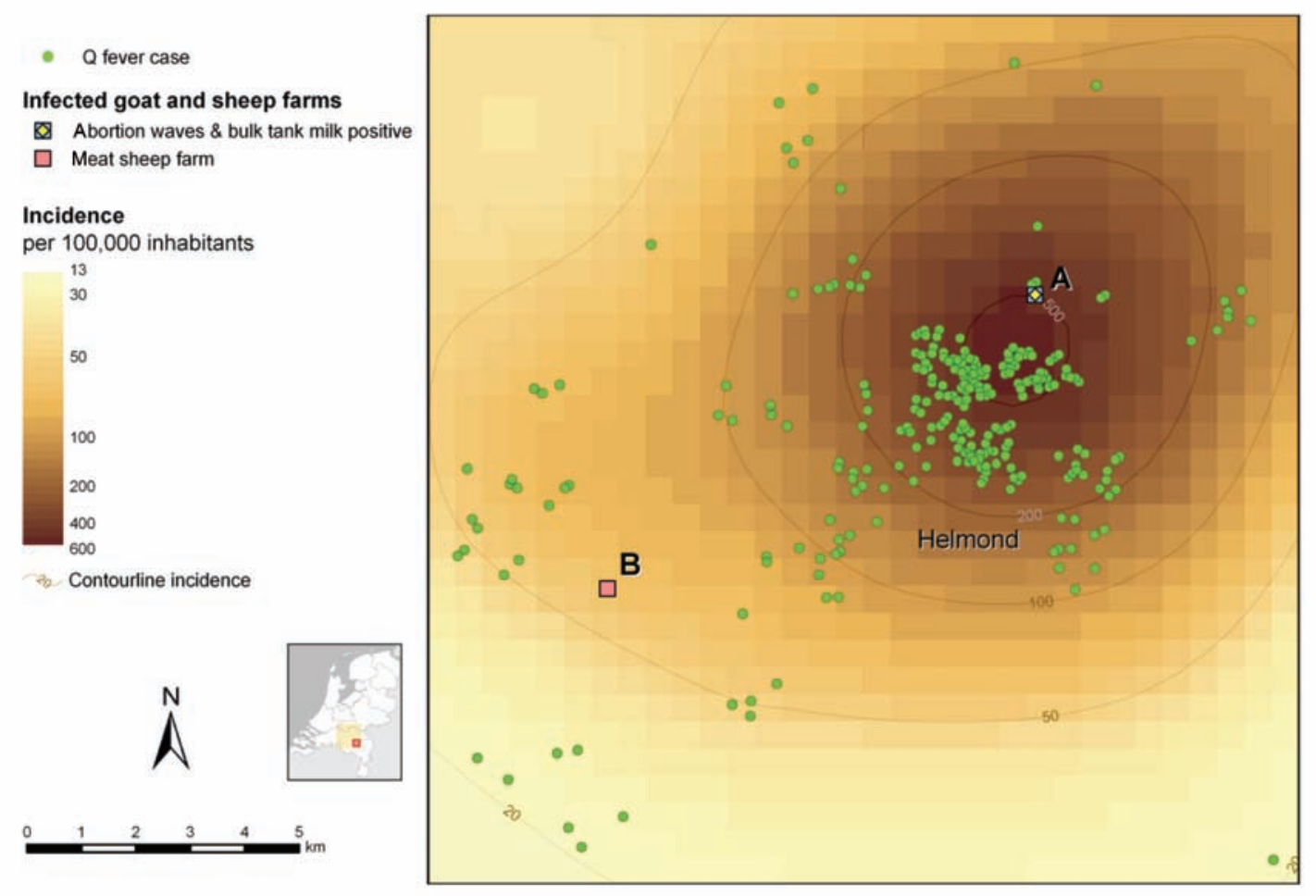

Fig. 4. Detailed smooth incidence map with the locations of farms A and B. 
Incidence maps are commonly presented by administrative areas, for which population figures are publicly available. Such maps are useful as an indication of epidemic spread and for information about health policy. However, for source detection of diseases with environmental determinants, administrative areas are not relevant because the risk may be biased by population density, e.g. underestimated in areas with low numbers of people and overrated in highly populated areas. We have shown that spatial statistical methods can be used to construct smooth incidence maps based on routine surveillance data. These maps have played an important role in decisions by public health and veterinary authorities on control measures with respect to Q fever. We also applied the method for Q fever outbreaks in the provinces of Utrecht (BrandsenSchreijer et al., 2010) and Limburg (unpublished) to inform public health policy. The method is automated and could rapidly be deployed for outbreaks of other infectious diseases in which environmental sources are suspected to play a role. It provides the opportunity to detect and investigate disease clusters and point to possible environmental sources to inform local public health departments.

The procedure is closely related to other methods of estimating intensity surfaces of spatial point processes. "Griddifying" areas is comparable to that of the logGaussian Cox process (Møller and Waagepetersen, 2003), except that our estimation procedure involves a smoothing parameter. Two-dimensional P-spline smoothing of Poisson data has already been successfully applied to smooth and extrapolate large mortality tables (Currie et al., 2006). Although all matrices are fairly large, they are extremely sparse, so fast computational techniques can be applied (Bates and Maechler, 2012). A map can be created by a fully automatic process in only a few seconds. If a measure of uncertainty is required, the standard errors for the $\log$ incidence estimates $\eta$ are obtained by taking the square root of $\operatorname{diag}\left\{\left(B^{T} W B+\lambda P\right)^{-1}\right\}$.

The smooth incidence maps are based solely on locations of the home address of human cases, and the populations at risk are treated as spatial point patterns. This assumes that human Q fever incidence is adequately represented by address (postal code) at the time of notification, thereby ignoring mobility of people with possible exposure in the workplace or elsewhere. The method described should be considered as an exploratory search for human Q fever hotspots and potential infection sources based on visual inspection of maps. It shows that even without formal statistical analysis it is possible to obtain important information.
More advanced statistical methods can be applied to extend the analyses. For an epidemiological risk factor analysis and causal inference, the method would have to be extended with information on relevant covariates for the human cases, such as age, sex, work history, work address, outdoor recreational activities, travel history and smoking habits. A proper exposure assessment would also have to include more detailed information on infected farms, e.g. the period they are infective. While not included in the present analyses, the method of smooth incidence maps can be extended to include temporal aspects. We have not accounted for clustering within households. However, humanto-human transmission of $\mathrm{Q}$ fever is extremely rare (Maurin and Raoult, 1999) and any clustering within households or in small geographical areas must be due to a common point source.

Accuracy of the diagnostic procedure, availability of laboratory facilities and awareness may play a role and could potentially bias the outcome. In 2009, being the third year of the epidemic, there was an increased awareness among the public and treating physicians to consider $\mathrm{Q}$ fever in their diagnostic agenda. However, although laboratory facilities have improved, diagnosing acute $\mathrm{Q}$ fever has become more difficult due to increasing seroprevalence with persistence of markers of acute infection, which may have caused overestimation of acute cases in this period.

\section{Conclusion}

Smooth incidence maps of human Q fever can rapidly document the development of epidemics and should therefore be considered when public health policies are established. Such maps are based on exploratory analysis of simple human and veterinary surveillance data and could be used for other infectious diseases in which human-to-human transmission does not play an important role. The present analysis supports previous observations that most human $\mathrm{Q}$ fever cases during the outbreaks in The Netherlands were caused by environmental exposure from infected dairy goat farms.

\section{Acknowledgements}

We thank the municipal health services "Hart voor Brabant" and "Rivierenland" for making available the postal codes of notified Q fever cases. This study was financed from the regular budget of the Centre for Infectious Disease Control made available by the Ministry of Health, Welfare and Sport, project numbers V/210231/01 and S/210106/01. 


\section{References}

Astobiza I, Barandika JF, Ruiz-Fons F, Hurtado A, Povedano I, Juste RA, García-Pérez AL, 2011. Coxiella burnetii shedding and environmental contamination at lambing in two highly naturally-infected dairy sheep flocks after vaccination. Res Vet Sci 91, e58-e63.

Bates D, Maechler M, 2012. Matrix: sparse and dense matrix classes and methods. R package version 1.0-3. Available at: http://cran.r-project.org/web/packages/Matrix/index.html (accessed on January 2012).

Brandsen-Schreijer AJM, Rump BO, Schimmer B, Cox CPGE, van den Bergh JPAM, Hulshof F, Hulshof K, van der Hoek W, Woonink F, 2010. Q fever outbreak in the province of Utrecht in the Netherlands in 2009. Infectiez Bull 21, 334-341.

Currie ID, Durban M, Eilers PHC, 2006. Generalised linear array models with applications to multidimensional smoothing. J R Statist Soc B 68, 259-280.

de Bruin A, de Groot A, de Heer L, Bok J, Hamans M, van Rotterdam BJ, Wielinga PR, Janse I, 2011. Detection of Coxiella burnetii in complex matrices by using multiplex qPCR during a major Q fever outbreak in the Netherlands. Appl Environ Microbiol 77, 6516-6523.

Dijkstra F, van der Hoek W, Wijers N, Schimmer B, Rietveld A, Wijkmans CJ, Vellema P, Schneeberger PM, 2012. The 20072010 Q fever epidemic in the Netherlands: characteristics of notified acute $\mathrm{Q}$ fever patients and the association with dairy goat farming. FEMS Immunol Med Microbiol 64, 3-12.

Eilers PHC, Marx DB, 1996. Flexible smoothing with B-splines and penalties (with comments and rejoinder). Stat Sci 11, 89121.

Eilers PHC, Marx BD, 2010. Splines, knots, and penalties. WIREs Comput Stat 2, 637-653.

Gilsdorf A, Kroh C, Grimm S, Jensen E, Wagner-Wiening C, Alpers K, 2005. Large Q fever outbreak due to sheep farming near residential areas, Germany, 2005. Epidemiol Infect 136, 1084-1087.

Koene RP, Schimmer B, Rensen H, Biesheuvel M, de Bruin A, Lohuis A, Horrevorts A, Lunel FV, Delsing CE, Hautvast JL, 2011. A Q fever outbreak in a psychiatric care institution in the Netherlands. Epidemiol Infect 139, 13-18.

Lawson A, 2006. Statistical methods in spatial epidemiology. $2^{\text {nd }}$ edition. Chichester, John Wiley \& Sons.

Lewin-Koh NJ, Bivand R, 2012. Maptools: tools for reading and handling spatial objects. $\mathrm{R}$ package version 0.8-14. Available at: http://cran.r-project.org/web/packages/maptools /index.html (accessed on January 2012).
Maurin M, Raoult D, 1999. Q fever. Clin Microbiol Rev 12, 518-553.

Møller J, Waagepetersen R, 2003. Statistical inference and simulation for spatial point processes. Volume 100 of Monographs on Statistics and Applied Probability. Chapman and Hall, London, UK.

Porten K, Rissland J, Tigges A, Broll S, Hopp W, Lunemann M, van Treeck U, Kimmig P, Brockmann SO, Wagner-Wiening C, Hellenbrand W, Buchholz U, 2006. A super-spreading ewe infects hundreds with $\mathrm{Q}$ fever at a farmers' market in Germany. BMC Infect Dis 6, 147.

R Development Core Team, 2012. R: a language and environment for statistical computing. Vienna, Austria. R Foundation for Statistical Computing. Available at: http://www.r-project.org/ (accessed on January 2012).

Roest HIJ, Tilburg JJHC, van der Hoek W, Vellema P, van Zijderveld FG, Klaassen CHW, Raoult D, 2011. The Q fever epidemic in the Netherlands: history, onset, response and reflection. Epidemiol Infect 139, 1-12.

Schimmer B, Dijkstra F, Vellema P, Schneeberger PM, Hackert V, ter Schegget R, Wijkmans C, van Duynhoven Y, van der Hoek W, 2009. Sustained intensive transmission of Q fever in the south of the Netherlands, 2009. Euro Surveill 14, pii 19210.

Schimmer B, Morroy G, Dijkstra F, Schneeberger PM, WeersPothoff G, Timen A, Wijkmans C, van der Hoek W, 2008. Large ongoing $\mathrm{Q}$ fever outbreak in the south of the Netherlands, 2008. Euro Surveill 13, pii 18939.

Schimmer B, Ter Schegget R, Wegdam M, Züchner L, de Bruin A, Schneeberger PM, Veenstra T, Vellema P, van der Hoek W, 2010. The use of a geographic information system to identify a dairy goat farm as the most likely source of an urban Q-fever outbreak. BMC Infect Dis 10, 69.

van der Hoek W, Dijkstra F, Schimmer B, Schneeberger PM, Vellema P, Wijkmans C, ter Schegget R, Hackert V, van Duynhoven Y, 2010. Q fever in the Netherlands: an update on the epidemiology and control measures. Euro Surveill 15 pii 19520.

van der Hoek W, Hunink J, Vellema P, Droogers P, 2011. Q fever in the Netherlands: the role of local environmental conditions. Int J Environ Health Res.

Whelan J, Schimmer B, de Bruin A, Robert-Du Ry van Beest Holle M, van der Hoek W, ter Schegget R, 2012. Visits on "lamb-viewing days" at a sheep farm open to the public was a risk factor for Q fever in 2009. Epidemiol Infect 140, 858-864. Wood SN, 2006. Generalised Additive Models: An Introduction with R. Chapman and Hall, CRC. London, UK. 